\title{
Microgravity and Telomeres
}

\author{
Dr. Roberto Aquilano \\ Facultad de Ciencias Exactas, Ingeniería y Agrimensura, Universidad Nacional de Rosario (UNR), \\ Av. Pellegrini 250, 2000 Rosario, Argentina
}

\begin{abstract}
Variations in telomeres, and especially their shortening, is a well-known process that marks the rhythm of life in all living things, but the reason behind telomere lengthening in some cases is not so well understood. We only know that it is directly related to the activation of an enzyme called telomerase. Variations in microgravity could partially explain this phenomenon, and be part of the cause of its activation.
\end{abstract}

Keywords - Microgravity; Telomeres; Telomerase; Space

\section{Introduction}

Almost all the laws of physics are symmetric in time, and the classic and most obvious solution is the thermodynamic arrow of time. Entropy in the field of information theory, called information entropy or Shannon entropy [1,2], measures the uncertainty of a source of information. Entropy is conceived as a measure of disorder, but it can also be considered as a measure of uncertainty and the information necessary to limit, reduce or eliminate uncertainty in any process.

The concepts of entropy and information are basically interrelated, although it took years of development in statistical mechanics and information theory before this could become apparent. Entropy in information theory is closely linked with entropy in thermodynamics.

The interaction between particles, in a closed system, tends to increase their diffusion, which affects their positions and their velocities, and increases the entropy of the distribution over time until a certain maximum is reached, which is known as the second law of thermodynamics. The difference between the amount of entropy in a system and its maximum potential is called negentropy and represents the amount of internal organization in the system.

Since the fundamental laws of physics are reversible at any time, it can be argued that irreversibility in thermodynamics must be of a statistical nature, which means that it must be very unlikely, but not impossible. We have the feeling that time flows from the past to the future, and that time has a fixed direction towards a future of entropy and growing disorder. However, in the microscopic world, this is not necessarily so. As we saw in previous research [3-6], everything seems clear at the macroscopic level, but at the microscopic level, it is more difficult to say that entropy is increasing and, therefore, time is progressing.

\section{Description and theory}

Today, thanks to advances in technology, and fundamentally in biotechnology, it is possible to study individual biological molecules. The high sensitivity of the optical tweezers, for example, allows subnanometric movements and rotations.

Using these techniques, Feng and Crooks [7] created a method to accurately measure time skewness at the microscopic level. Thanks to a new measurement or evaluation method, they showed that time seems to move into the future even when entropy decreases. For this, they used experimental data [8,9] to analyze the folding and unfolding of an RNA molecule supported by its ends and found that, in certain intervals, entropy can decrease. Although overall entropy increases on average, time does not always have a clear direction.

They were able to demonstrate that, at certain intervals, entropy can actually decrease. Therefore, we can conclude that the direction of time is not clear at the scale of a single molecule.

They took a capture with an optical laser trap that could measure the applied force. The RNA was initially in thermal equilibrium while twisting. In the time reversal protocol, the RNA was initially in thermal equilibrium during development, and then shortened and allowed the RNA to fold. In these experiments, a mechanical force is applied to both ends of the molecule, and the mechanical properties of the molecule can be analyzed with this technique.

The value of the applied force basically grows linearly until the molecule unfolds. If the process is reversed, relaxing the tension applied in the system, the molecule folds back on itself. The information obtained from these experiments is the force as a function of the distance from end to end of the system. The entangled molecules unravel 
very slowly, and it was shown that this process generates a large dispersion or average increase in entropy and a small temporal asymmetry, as would be expected intuitively due to slow traction.

\section{Discussion}

The proposed approach is whether this same mechanism could be related to what happens to astronauts in space, where the effect of microgravity variations could produce a mechanism equivalent to what Feng and Crooks achieved in the laboratory, causing the activation of the telomerase enzyme, and producing the lengthening of telomeres as happened to astronaut Scott Kelly, who spent almost a year in space. This investigation by Susan Bailey [8] could be an alternative mechanism to explain a phenomenon that remained unexplained until now.

In addition, we now know a lot about how tumor cells stop the aging process by stretching telomeres [9,10], and that cells that have undergone a transformation usually have telomerase activity that is repressed in adult cells and with each division telomeres they shorten.

Everything points to the telomeres involved in differentiated cells that have an unlimited number of cell divisions, after which they die or stop dividing in a process known as cellular senescence. Telomere shortening is related to the replicative senescence of differentiated somatic cells that lack telomerase activity, which is thought that telomeric shortening works like a clock that counts the number of cell divisions left in certain cells.

Telomerase ensures that telomeres remain in optimal conditions in the next generation, but disappears after birth, causing telomeres to shorten and the aging process begins. When telomerase returns, telomeres grow back and can become a problem if the process is not controlled. If the treated cells begin to divide, they can become very dangerous because cancer is very likely to develop in the process.

After spending 340 days in space at the International Space Station (ISS), astronaut Scott Kelly presented changes in his gene expression, DNA methylation and other biomarkers. Like identical twins, Scott and Mark Kelly are genetically very similar. However, it was discovered that while Scott was in orbit, the ends of his chromosomes grew until they became longer than his brother's, although they quickly regained their original length shortly after returning to Earth. This was a completely unexpected discovery.

Telomeres tend to shorten naturally as we age and it was assumed that the rigors of space would increase DNA damage, especially radiation, and accelerate shortening, and not vice versa. The most amazing transformation observed so far in Scott Kelly's body is precisely in his DNA, and specifically in his telomeres, the ends of the chromosomes. This could be due to the activation of telomerase as a result of space travel and associated environmental variables, such as exposure to low doses of radiation or the absence of gravity. Clarifying the molecular mechanisms underlying these observations will be of interest to understand how space affects the length of telomeres and, therefore, the body's regenerative ability to plan future manned space travel.

\section{Conclusion}

Apparently, as we see, time does not have a clear direction, and the asymmetry of time is not certain, so in the microscopic world the phenomenon would not be like in the macroscopic in some cases. Furthermore, when comparing these experiments with the conditions suffered by astronauts in space, we can assume that variations in microgravity could act similarly in the aforementioned experiments, so this mechanism could become one of the explanations for activation of telomerase in space.

We are currently planning experiments to send humans, mice, and plants into space, to perform them on microgravity platforms, and on the International Space Station. In this way we think of studying the effects of variations in microgravity on telomeres to see if it is the cause of the activation of the mentioned enzyme.

\section{Acknowledgments}

I wish to thank to María Blasco Maruhenda of National Cancer Centre (CNIO) of Spain and Susan Bailey of Department of Environmental and Radiological Health Sciences of Colorado State University of and of NASA's Twin Study of USA, for the support received and their useful discussions. As well as Ana Caño Delgado from CRAG-CSIC of Spain, Emmanuel Lalla, from York University in Canada, Daniel Portnoy from Tel Aviv University and Shimon Amselem from the Space Pharma company in Israel.

\section{References}

[1] Shannon C.E. (1948) A mathematical theory of communication, Bell System Technical Journal 27 (379-423 and 623-656)

[2] Shannon C.E. (1949) Communication theory of secrecy systems, Bell System Technical Journal 28 (656-715)

[3] Aquilano R. (2017) Austin Biochem., 2, 1, 1008

[4] Aquilano R. (2017) Austin Biochem. 2, 2, 1011

[5] Aquilano R. and Blasco M. (2018), Austin Biochem. 3, 1, 1019

[6] Aquilano R. (2019), Ad. Stud. Theor. Phys. 13, 8, 379-386

[7] Feng E.H., Crooks G E (2008) Phys. Rev. Lett. 101, 090602

[8] Garrett-Bakelman F.E. et al. (2019) Science 364, eaau8650

[9] Tomas-Loba A. et al. (2008) Cell 609, 22

[10] Blackburn, E. (2001), Cell 106, 661 